\title{
Convergence of linear barycentric rational interpolation for analytic functions
}

\author{
Stefan Güttel* Georges Klein ${ }^{\dagger}$
}

\begin{abstract}
Polynomial interpolation to analytic functions can be very accurate, depending on the distribution of the interpolation nodes. However, in equispaced nodes and the like, besides being badly conditioned, these interpolants fail to converge even in exact arithmetic in some cases. Linear barycentric rational interpolation with the weights presented by Floater and Hormann can be viewed as blended polynomial interpolation and often yields better approximation in such cases. This has been proven for differentiable functions and indicated in several experiments for analytic functions. So far, these rational interpolants have been used mainly with a constant parameter usually denoted by $d$, the degree of the blended polynomials, which leads to small condition numbers but to merely algebraic convergence. With the help of logarithmic potential theory we derive asymptotic convergence results for analytic functions when this parameter varies with the number of nodes. Moreover, we present suggestions for how to choose $d$ in order to observe fast and stable convergence, even in equispaced nodes where stable geometric convergence is provably impossible. We demonstrate our results with several numerical examples.
\end{abstract}

Math Subject Classification: 65D05, 65E05, 41A20

Keywords: barycentric rational interpolation, potential theory, level curves, analyticity

\section{Introduction}

The rational approximation procedure we are concerned with is the following. On an interval $[a, b]$ of the real line, a function $f$, which is assumed to be analytic in a neighborhood of $[a, b]$, is sampled at an ordered set of $n+1$ distinct nodes $a \leq x_{0, n}<x_{1, n}<\cdots<x_{n, n} \leq$

*Mathematical Institute, University of Oxford, Oxford OX1 3LB, UK (stefan.guettel@maths.ox.ac.uk). Current address: School of Mathematics, University of Manchester, Oxford Road, Manchester M13 9PL, UK. This author's work was supported by Deutsche Forschungsgemeinschaft Fellowship GU 1244/1-1.

${ }^{\dagger}$ Department of Mathematics, University of Fribourg, Pérolles, 1700 Fribourg, Switzerland (georges.klein@unifr.ch). This author's work was partially supported by the Swiss National Science Foundation under Grant 200020-135319. 
$b$, and we want to approximate $f$ on this interval by an analytic barycentric rational interpolant $r_{n}$, i.e.,

$$
r_{n}\left(x_{i, n}\right)=f\left(x_{i, n}\right)=: f_{i, n}, \quad i=0, \ldots, n,
$$

with $r_{n}$ having no poles in $[a, b]$. A family of linear barycentric rational interpolants with these properties has been presented by Floater and Hormann in [11]. They choose an integer parameter $0 \leq d \leq n$ and approximate $f$ by a "blend" of polynomials,

$$
r_{n}(x)=\frac{\sum_{i=0}^{n-d} \lambda_{i, n}(x) p_{i, n}(x)}{\sum_{i=0}^{n-d} \lambda_{i, n}(x)},
$$

where $p_{i, n}$ is the polynomial of degree $\leq d$ interpolating $f_{i, n}, \ldots, f_{i+d, n}$, and

$$
\lambda_{i, n}(x)=\frac{(-1)^{i}}{\left(x-x_{i, n}\right) \cdots\left(x-x_{i+d, n}\right)} .
$$

The original form of $r_{n}$ on the right-hand side of (1) is not well suited for numerical evaluation. Floater and Hormann established the barycentric form

$$
r_{n}(x)=\sum_{i=0}^{n} \frac{w_{i, n}}{x-x_{i, n}} f_{i, n} / \sum_{i=0}^{n} \frac{w_{i, n}}{x-x_{i, n}}
$$

and gave explicit formulas for the barycentric weights $w_{i, n}$, which do not depend on the data values $f_{0, n}, \ldots, f_{n, n}$. The rational function $r_{n}$ is thus linear in the data. Further details on barycentric rational interpolation and its implementation can be found in [3].

The family of rational interpolants (1) includes polynomial interpolation as a special case: $r_{n}$ simplifies to $p_{0, n}=: p_{n}$ if $d=n$. It is well known that polynomial interpolants may converge or diverge geometrically fast, depending on the distribution of the nodes and the domain of analyticity of the interpolated function. To make this statement more precise, we take advantage of some notions and a result on polynomial interpolation from [12] (see also [22]). Let the interpolation nodes $x_{i, n}$ be distributed in $[a, b]$ according to a probability measure $\mu$ with support $[a, b]$ and positive piecewise continuous node density

$$
\phi(x)=\frac{\mathrm{d} \mu}{\mathrm{d} x}(x)>0 \quad \text { for } x \in[a, b] .
$$

This statement can be made precise by defining the normalized discrete measures

$$
\mu_{n}=\frac{1}{n+1} \sum_{i=0}^{n} \delta_{x_{i, n}}
$$

where $\delta_{x}$ denotes the Dirac unit measure at $x$. We require that $\mu_{n} \rightarrow \mu$, in the sense of weakstar convergence of measures, which means that $\int g \mathrm{~d} \mu_{n} \rightarrow \int g \mathrm{~d} \mu$ for every continuous function $g$ defined on $[a, b]$. Associated with the limiting measure $\mu$, which we will also refer to as the node measure, is a logarithmic potential

$$
U^{\mu}(z):=-\int_{a}^{b} \log |z-x| \mathrm{d} \mu(x)=-\int_{a}^{b} \phi(x) \log |z-x| \mathrm{d} x .
$$


By the conditions on the density of $\mu$, the real-valued function $U^{\mu}$ is harmonic in the complex plane $\mathbb{C}$ and decays like $-\log |z|$ as $|z| \rightarrow \infty$. The convergence or divergence of a sequence of polynomial interpolants $p_{n}$ for an analytic function $f$ is then described by the following classical theorem (see, e.g., [12]).

Theorem 1 For a given node measure $\mu$ and the associated potential $U^{\mu}$, let $f$ be analytic inside $\mathcal{C}_{s}$, the level line of $U^{\mu}$ which passes through a singularity $s$ of $f$. The polynomial interpolant $p_{n}$ of $f$ then converges to $f$ inside $\mathcal{C}_{s}$ and diverges outside, and

$$
\lim _{n \rightarrow \infty}\left|f(z)-p_{n}(z)\right|^{1 / n}=\exp \left(U^{\mu}(s)-U^{\mu}(z)\right) .
$$

In the special case of equispaced nodes, i.e., if $\mathrm{d} \mu / \mathrm{d} x=1 /(b-a)$, the potential becomes [12, section 3.4]

$$
U_{\mathrm{eq}}^{\mu}(z)=-\log \left(\frac{b-a}{2 e}\right)-\frac{1}{2} \operatorname{Re}\left(\left(1-z^{\prime}\right) \log \left(1-z^{\prime}\right)-\left(-1-z^{\prime}\right) \log \left(-1-z^{\prime}\right)\right),
$$

where $z^{\prime}=(2 z-a-b) /(b-a)$.

The Chebyshev extrema $x_{i, n}=-\cos (i \pi / n)$, which we will also refer to as Chebyshev points, are distributed according to the density function $\phi(x)=1 /\left(\pi \sqrt{1-x^{2}}\right)$, and the associated potential is

$$
U_{\mathrm{Ch}}^{\mu}(z)=-\int_{-1}^{1} \frac{\log |z-x|}{\pi \sqrt{1-x^{2}}} \mathrm{~d} x=-\log \left|z+\sqrt{z} \cdot \sqrt{z-z^{-1}}\right|+\log (2),
$$

which turns out to be constant on the interval $[-1,1]$. The associated node measure $\mu$, with the property that there is no potential difference on $[-1,1]$, is the so-called equilibrium measure. Interpolation nodes which are distributed according to that measure are, in an asymptotic sense, optimal for polynomial interpolation.

In Figure 1 we have plotted the asymptotic rates of convergence or divergence of polynomial interpolation on $[-1,1]$ with equispaced nodes (on the left) and Chebyshev points (on the right) as a function of $s$. To be more precise, for each $s$ in $(-2,2) \times(-2 \mathrm{i}, 2 \mathrm{i})$, we took the maximum over $[-1,1]$ of the expression on the right-hand side of (5). The picture on the left shows that polynomial interpolation with equispaced nodes does not converge throughout $[-1,1]$ if $f$ has a singularity $s$ too close to the interval. On the other hand, the picture on the right shows that polynomial interpolation with Chebyshev points converges throughout the interval.

This theory gives an explanation of Runge's phenomenon [10, 21]. Polynomial interpolation in equispaced nodes on $[-1,1]$ for the Runge example $1 /\left(1+25 x^{2}\right)$ does not converge on the whole interval. This is explained by the fact that the level line of $U_{\mathrm{eq}}^{\mu}$ passing through the singularities $s= \pm \mathrm{i} / 5$ does not enclose $[-1,1]$ but only $[-0.7266,0.7266]$, as can be seen on the left picture in Figure 1. The Runge phenomenon appears in the form of oscillations toward the ends of the interval. The level line of $U_{\mathrm{Ch}}^{\mu}$ that passes through $s= \pm \mathrm{i} / 5$ includes the interval $[-1,1]$; see the right part of Figure 1. The phenomenon therefore does not appear when interpolating a function, such as that given above, in Chebyshev 

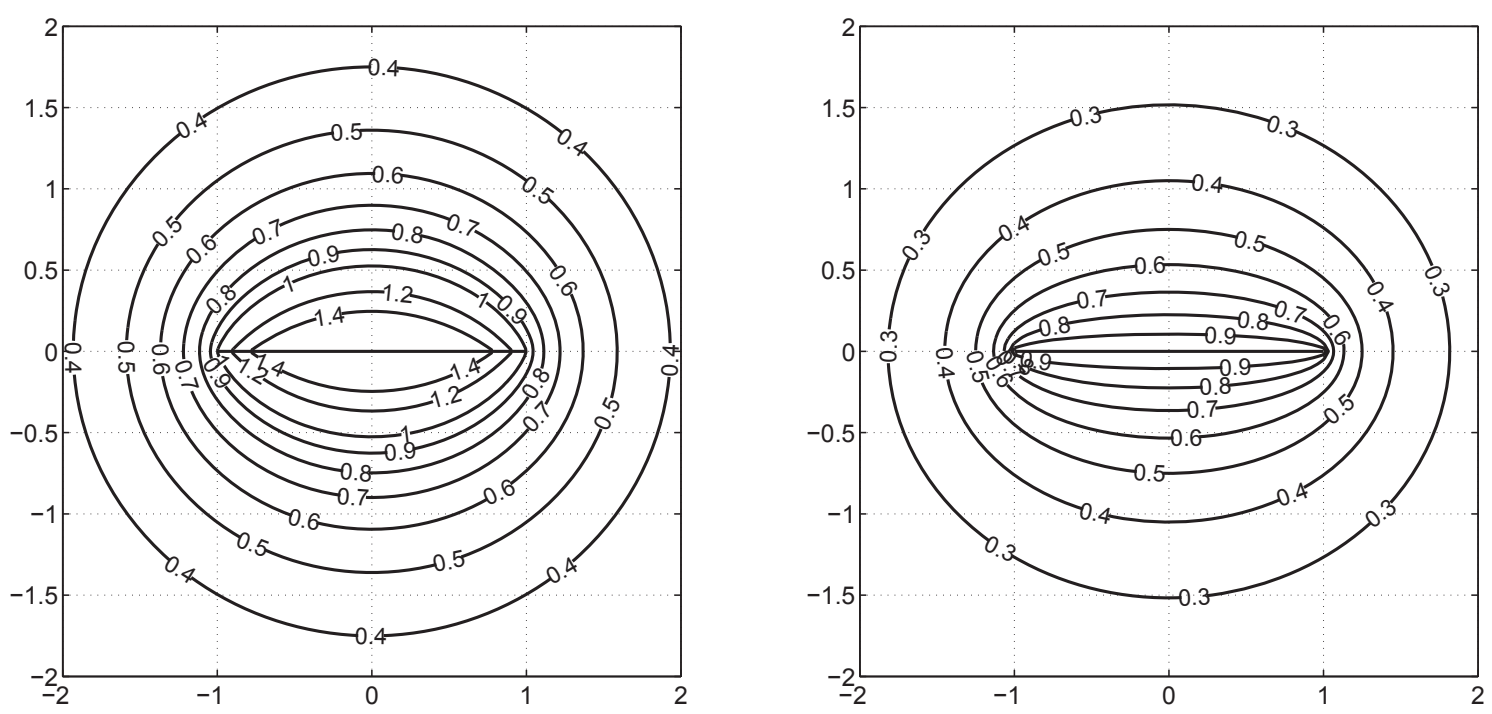

Figure 1: Level lines of $\exp \left(U^{\mu}(s)-\min _{-1 \leq x \leq 1} U^{\mu}(x)\right)$ in the complex plane for polynomial interpolation in equispaced nodes (left) and in Chebyshev points (right). The central line represents the interval $[-1,1]$.

points and other nodes with quadratic clustering toward the ends of the interval. These polynomial interpolants converge geometrically fast as the number of nodes increases.

Convergence results for analytic functions, based on potential theory as in Theorem 1, have also been derived for other interpolation schemes, such as linear barycentric rational interpolation in mapped Chebyshev points [1], radial basis functions [16, 17], and nonlinear rational interpolants $[25,30,31]$. Such a theory does not yet exist for the family of barycentric rational interpolants (1). Our aim is to fill this gap with Theorem 2 in the next section. The authors of [11] proved a convergence result for differentiable functions, namely, that for any function $f$ in $C^{d+2}[a, b]$,

$$
\left\|f-r_{n}\right\|_{\infty}=\max _{a \leq x \leq b}\left|f(x)-r_{n}(x)\right| \leq K h^{d+1},
$$

where $h=\max _{0 \leq i \leq n-1}\left(x_{i+1, n}-x_{i, n}\right)$ and $K$ is a constant depending only on $d$ and on derivatives of $f$. For $d$ fixed, this error bound indicates that Runge's phenomenon does not occur. In exact arithmetic, the rational interpolants converge algebraically to $f$ as $n$ increases, provided the function is sufficiently smooth, even if it has singularities very close to $[a, b]$.

Besides the convergence results in exact arithmetic, there is the numerical error, which, additionally, might have been influenced by rounding errors or noise in the data values. The condition number of linear barycentric interpolation is equal to its Lebesgue constant

$$
\Lambda_{n}=\max _{a \leq x \leq b} \Lambda_{n}(x)=\max _{a \leq x \leq b} \sum_{i=0}^{n}\left|\frac{w_{i, n}}{x-x_{i, n}}\right| /\left|\sum_{i=0}^{n} \frac{w_{i, n}}{x-x_{i, n}}\right|,
$$



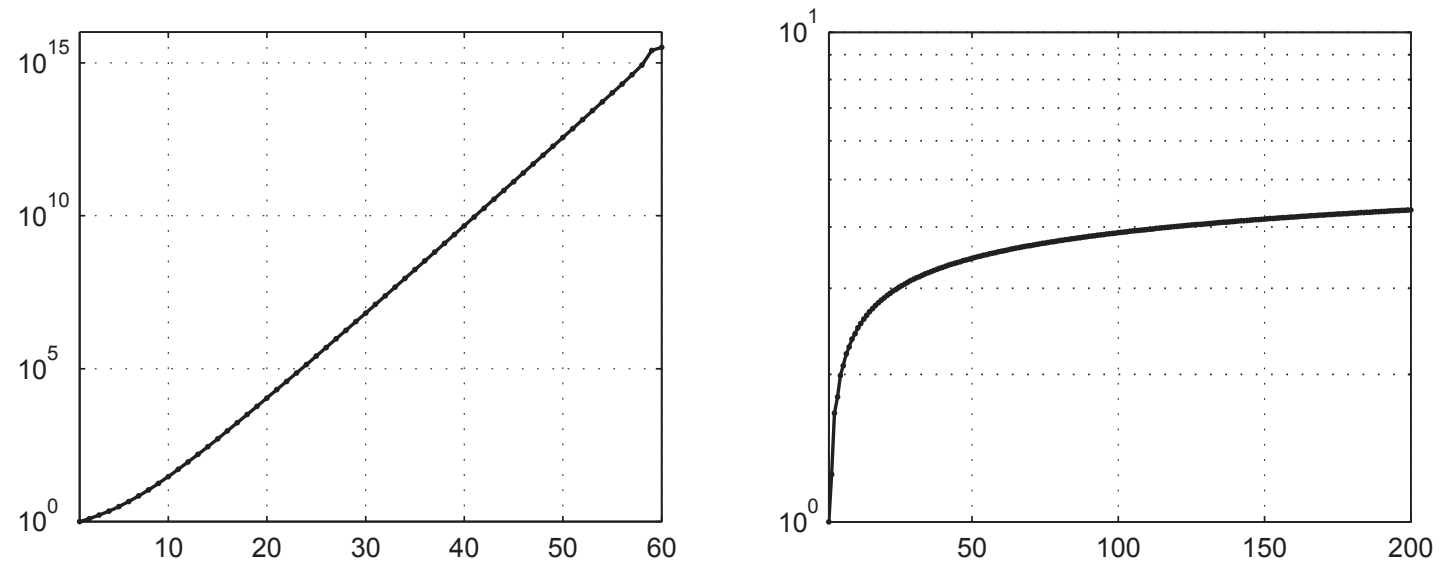

Figure 2: Lebesgue constants associated with polynomial interpolation in $1 \leq n \leq 60$ equispaced nodes (left) and in $1 \leq n \leq 200$ Chebyshev points (right).

which is the maximum of the Lebesgue function $\Lambda_{n}(x)$. This represents the maximal amplification of any imprecision in the data $f_{0, n}, \ldots, f_{n, n}$ during the interpolation process. For the family $r_{n}$ from (2), it is shown in $[4,5,13]$ that $\Lambda_{n}$ grows logarithmically with $n$ and geometrically with $d$ for equispaced and quasi-equispaced nodes. By the latter we mean that $h / h_{*}$, with $h_{*}=\min _{0 \leq i \leq n-1}\left(x_{i+1, n}-x_{i, n}\right)$, is bounded by a constant $M$ independent of $n$; see [13]. For Berrut's rational interpolant [2], that is, $r_{n}$ with $d=0$, the Lebesgue constant also grows only logarithmically as $n$ increases with more general nodes, generated from an inverse cumulative node distribution $\Phi^{-1}$ from (9) satisfying a certain regularity condition; see [6] and section 2.1. In the case of equispaced nodes and $d \geq 1$, the following upper bound has been established:

$$
\Lambda_{n} \leq 2^{d-1}(2+\log n)
$$

For $d=0$, the factor $2^{d-1}$ in the above bound may be replaced by $3 / 4$; see [13, Thm. 2.2].

The Lebesgue constant associated with polynomial interpolation in Chebyshev points grows very slowly with $n$; see $[7,9,24]$. This property, together with numerous additional facts, makes the combination of polynomial interpolation with Chebyshev points a very successful approximation procedure that is useful for many applications such as Chebfun $[26,27]$. In equispaced nodes, however, polynomial interpolation and any other approximation procedure with geometric convergence yield condition numbers that grow geometrically fast as the number of nodes increases [18, 23, 28, 29]. Figure 2 illustrates these facts. The curve in the left picture, showing Lebesgue constants associated with polynomial interpolation in equispaced nodes, is very similar to that obtained for Lebesgue constants associated with the Floater-Hormann family in equispaced nodes with $n \geq 60$ fixed and $0 \leq d \leq 60$. The picture on the right of Figure 2 represents the logarithmic growth of the Lebesgue constants associated with polynomial interpolation in Chebyshev points. 
In section 2, we establish our main asymptotic convergence theorem for general node densities and treat the special cases of symmetric nodes and equispaced nodes, for which further insight can be given. Section 3 presents some suggestions about the choice of $d$ depending on the number of nodes and the analyticity of $f$ to find a good compromise between fast convergence and high condition numbers. Finally, we present a few experimental results in section 4 .

\section{Asymptotic convergence analysis}

The rational interpolants (1) are blends of the local polynomial interpolants $p_{i, n}$, and we use this fact in the derivation of an asymptotic upper bound on the interpolation error for analytic functions. We begin with some statements about the node densities of the subsets $\left\{x_{i, n}, \ldots, x_{i+d, n}\right\}$, which are the interpolation nodes of $p_{i, n}$. We then develop the main convergence result of the present paper. The end of this section is concerned with the important special cases of symmetric nodes and equispaced nodes, for which we further expand the expression of the bound on the interpolation error and give additional insight into the convergence of the interpolants.

\subsection{General node densities}

We assume from now on that the parameter $d$, defining a particular rational interpolant from the family (1), is a variable non-negative integer $d(n)$ such that

$$
d(n) / n \rightarrow C, \quad n \rightarrow \infty
$$

for a fixed $C \in(0,1]$. In practice, one could choose $d(n)=\operatorname{round}(C n)$. By the positivity of $\phi$ from (3), the cumulative node distribution

$$
\Phi(x):=\mu([a, x])=\int_{a}^{x} \phi(x) \mathrm{d} x
$$

is a continuous and strictly monotonically increasing function on the interval $[a, b]$ and therefore has a continuous inverse

$$
\Phi^{-1}:[0,1] \rightarrow[a, b]
$$

With this definition and that of the discrete measure $\mu_{n}$ from (4), we have the following simple result, which we state as a lemma for easier reference.

Lemma 1 Assume that $\mu_{n} \rightarrow \mu$ as $n \rightarrow \infty$, where the limit measure $\mu$ has piecewise continuous positive density, and let $x_{j(n), n}$ be an arbitrary sequence of nodes. Then $x_{j(n), n} \rightarrow$ $x$ for some $x$ if and only if there exists some $q \in[0,1]$ such that $(j(n)+1) /(n+1) \rightarrow q$. In this case, $x=\Phi^{-1}(q)$. 
Proof. Under the assumptions on $\mu$, the set $\{x\}$ is a continuity set of $\mu$ for every point $x \in[a, b]$. The condition that $\mu_{n} \rightarrow \mu$ thus implies that $\mu_{n}([a, x]) \rightarrow \mu([a, x])$. By the definition of $\mu_{n}$ and $\Phi$, this is equivalent to

$$
\lim _{n \rightarrow \infty} \frac{\#\left\{x_{i, n}: x_{i, n} \leq x\right\}}{n+1}=\Phi(x) .
$$

With $j(n):=\max \left\{0, \#\left\{x_{i, n}: x_{i, n} \leq x\right\}-1\right\}$ and $q:=\Phi(x)$ we obtain, as required,

$$
\lim _{n \rightarrow \infty} \frac{j(n)+1}{n+1}=q
$$

We now suppose that $j(n)$ is a sequence of indices such that $j(n) \leq n-d(n)$ and $x_{j(n), n} \rightarrow \alpha$ for some $\alpha \in[a, b]$. Under the condition (8) on $d(n)$ it follows from Lemma 1 that the sequence of nodes $x_{j(n)+d(n), n}$ also converges to a point in $[a, b]$, which we call $\beta(\alpha)$, and satisfies

$$
\lim _{n \rightarrow \infty} x_{j(n)+d(n), n}=\Phi^{-1}(C+\Phi(\alpha))=\beta(\alpha) .
$$

The nodes $x_{j(n), n}, \ldots, x_{j(n)+d(n), n}$ are therefore asymptotically contained in the interval $[\alpha, \beta(\alpha)]$, and they are distributed with density $\phi$ restricted to that interval. More precisely, the normalized discrete measures

$$
\nu_{j(n), n}=\frac{1}{d(n)+1} \sum_{i=0}^{d(n)} \delta_{x_{j(n)+i, n}}
$$

converge (in the weak sense) to a probability measure $\nu_{\alpha}$ with support $[\alpha, \beta(\alpha)]$ and density

$$
\frac{\mathrm{d} \nu_{\alpha}}{\mathrm{d} x}(x)=\frac{\phi(x)}{\Phi(\beta(\alpha))-\Phi(\alpha)}=\frac{\phi(x)}{C} .
$$

Note that $\nu_{\alpha}$ is simply the normalized restriction of $\mu$ to the interval $[\alpha, \beta(\alpha)]$. The study of the convergence of measures and their associated potentials is an important tool in logarithmic potential theory $[20,22]$. A main ingredient is the so-called principle of descent (see [22, Theorem I.6.8]): If $S$ is compact and $\left(\sigma_{n}\right)$ is a sequence of finite positive Borel measures with $\operatorname{supp}\left(\sigma_{n}\right) \subset S$, then

$$
\begin{aligned}
& \text { for } z_{n} \rightarrow z \text { and } \sigma_{n} \rightarrow \sigma, \quad \liminf _{n \rightarrow \infty} U^{\sigma_{n}}\left(z_{n}\right) \geq U^{\sigma}(z), \\
& \text { for } z_{n} \rightarrow z \notin S \text { and } \sigma_{n} \rightarrow \sigma, \quad \lim _{n \rightarrow \infty} U^{\sigma_{n}}\left(z_{n}\right)=U^{\sigma}(z) .
\end{aligned}
$$

These relations will allow us to take advantage of polynomial approximation theory for quantifying the convergence of the local interpolating polynomials $p_{j(n), n}$. The following two lemmas are a first step in this direction as they give asymptotic upper and lower bounds on the rational function

$$
\sum_{i=0}^{n-d(n)} \lambda_{i, n}(z),
$$

which appears in $r_{n}(z)$ and plays an important role in the subsequent analysis. 
Lemma 2 For any $C \in(0,1]$ and $z \in \mathbb{C} \backslash[a, b]$, we have

$$
\limsup _{n \rightarrow \infty}\left|\sum_{i=0}^{n-d(n)} \lambda_{i, n}(z)\right|^{1 /(n+1)} \leq \max _{\alpha \in\left[a, \Phi^{-1}(1-C)\right]} \exp \left(C U^{\nu_{\alpha}}(z)\right) .
$$

Proof. For every $n$ and every $z \in \mathbb{C} \backslash[a, b]$ we can find a dominating term $\lambda_{j, n}(z)$ with index $j=j(n)$ such that

$$
\left|\sum_{i=0}^{n-d(n)} \lambda_{i, n}(z)\right| \leq(n-d(n)+1)\left|\lambda_{j(n), n}(z)\right| .
$$

With a simple computation involving (10), we rewrite the second factor of the above righthand side:

$$
\begin{aligned}
\left|\lambda_{j(n), n}(z)\right| & =\prod_{i=0}^{d(n)}\left|z-x_{j(n)+i, n}\right|^{-1} \\
& =\exp \left(-\sum_{i=0}^{d(n)} \log \left|z-x_{j(n)+i, n}\right|\right) \\
& =\exp \left((d(n)+1) U^{\nu_{j(n), n}}(z)\right) .
\end{aligned}
$$

From the sequence of nodes $x_{j(n), n}$ we can select a subsequence, which we also denote by $x_{j(n), n}$, and which has the property that $x_{j(n), n} \rightarrow \alpha$ for $\alpha \in[a, b]$ fixed. Upon taking the $(n+1)$ st root and using the fact that $(d(n)+1) /(n+1) \rightarrow C$ as $n \rightarrow \infty$, we obtain that

$$
\limsup _{n \rightarrow \infty}\left|\sum_{i=0}^{n-d(n)} \lambda_{i, n}(z)\right|^{1 /(n+1)} \leq \limsup _{n \rightarrow \infty} \exp \left(C U^{\nu_{j(n), n}}(z)\right) .
$$

Since $z \notin[a, b]$ and $\nu_{j(n), n} \rightarrow \nu_{\alpha}$, it follows from the principle of descent that the limsup on the right-hand side equals $\exp \left(C U^{\nu_{\alpha}}(z)\right)$.

In the proof of the next lemma we reuse similar tools to derive a lower bound on the same function, but now evaluated at $x \in[a, b]$.

Lemma 3 For any $C \in(0,1]$ and $x \in[a, b]$, we have

$$
\liminf _{n \rightarrow \infty}\left|\sum_{i=0}^{n-d(n)} \lambda_{i, n}(x)\right|^{1 /(n+1)} \geq \max _{\substack{\alpha \in\left[a, \Phi^{-1}(1-C)\right] \\ x \in \operatorname{supp}\left(\nu_{\alpha}\right)}} \exp \left(C U^{\nu_{\alpha}}(x)\right) .
$$

Proof. We first suppose that $x \in\left(x_{k, n}, x_{k+1, n}\right)$. For every $n$ and every such $x$ it is shown in the proof of [11, Thm. 2] that

$$
\left|\sum_{i=0}^{n-d(n)} \lambda_{i, n}(x)\right| \geq\left|\lambda_{j, n}(x)\right|
$$


for all $j \in J:=\{i \in\{0,1, \ldots, n-d(n)\}: k-d(n)+1 \leq i \leq k\}$. On the other hand, if $x$ coincides with a node $x_{i, n}$, then this inequality is trivially valid (the left-hand side is $+\infty)$. For every $n$, we choose $j=j(n) \in J$ such that $\lambda_{j(n), n}(x)$ is the largest, and we form the corresponding sequence $j(n)$. From the sequence of nodes $x_{j(n), n}$ we select, as in the proof of Lemma 2, a subsequence such that $x_{j(n), n} \rightarrow \alpha$. Upon taking the $(n+1)$ st root on both sides of the above inequality, the principle of descent yields the asserted relation analogously as in the proof of Lemma 2 .

We are now prepared to investigate the asymptotic convergence of the family of rational interpolants (1). As it is explained, for instance, in [16, Thm. 3.2 and Corol. 3.4], it will be sufficient to investigate interpolants of "prototype functions" $g(x, s)=1 /(s-x)$ with a simple pole $s \in \mathbb{C} \backslash[a, b]$. An explicit expression for the local polynomial interpolants $p_{i, n}$ of such a particular function is

$$
p_{i, n}(x)=\frac{1-\frac{\lambda_{i, n}(s)}{\lambda_{i, n}(x)}}{s-x} .
$$

It is easily verified that this is indeed a polynomial satisfying $p_{i, n}\left(x_{j, n}\right)=1 /\left(s-x_{j, n}\right)$ for all nodes $x_{j, n}$ involved in $\lambda_{i, n}$. Hence, the rational interpolant (1) of $g$, which we denote by $r_{n}[g]$, is

$$
\begin{aligned}
r_{n}[g](x) & =\frac{1}{s-x} \cdot \frac{\sum_{i=0}^{n-d} \lambda_{i, n}(x)\left(1-\frac{\lambda_{i, n}(s)}{\lambda_{i, n}(x)}\right)}{\sum_{i=0}^{n-d} \lambda_{i, n}(x)} \\
& =\frac{1}{s-x} \cdot\left(1-\frac{\sum_{i=0}^{n-d} \lambda_{i, n}(s)}{\sum_{i=0}^{n-d} \lambda_{i, n}(x)}\right),
\end{aligned}
$$

and therefore

$$
g(x, s)-r_{n}[g](x)=\frac{1}{s-x} \cdot \frac{\sum_{i=0}^{n-d} \lambda_{i, n}(s)}{\sum_{i=0}^{n-d} \lambda_{i, n}(x)} .
$$

To make the subsequent notation more compact, we define the "potential function"

$$
V^{C, \mu}(z):= \begin{cases}\max _{\alpha \in\left[a, \Phi^{-1}(1-C)\right]} C U^{\nu_{\alpha}}(z), & z \in \mathbb{C} \backslash[a, b], \\ \max _{\alpha \in\left[a, \Phi^{-1}(1-C)\right]} C U^{\nu_{\alpha}}(z), & z \in[a, b] .\end{cases}
$$

Combining Lemmas 2 and 3, and using the monotonicity of the exponential function (note that the potentials $U^{\nu_{\alpha}}$ are real-valued functions), we finally arrive at

$$
\limsup _{n \rightarrow \infty}\left|g(x, s)-r_{n}[g](x)\right|^{1 / n} \leq \exp \left(V^{C, \mu}(s)-V^{C, \mu}(x)\right) .
$$

This statement closely resembles that from Theorem 1 and contains the latter as a special case: The rational interpolants reduce (asymptotically) to polynomial interpolants if $C=1$, 
and in this case the function $V^{C, \mu}$ in (13) reduces to $U^{\mu}$. Moreover, the potential $V^{C, \mu}(z)$ is a continuous function when the condition " $z \in \operatorname{supp}\left(\nu_{\alpha}\right)$ " in the second case of (13) is redundant, which is the case for any reasonable node measure $\mu$.

The uniform convergence over the whole interval $[a, b]$ is often of major interest in approximation theory. To establish such a result for the rational interpolants (1), we define the contours

$$
\mathcal{C}_{R}:=\left\{z \in \mathbb{C}: \frac{\exp \left(V^{C, \mu}(z)\right)}{\min _{x \in[a, b]} \exp \left(V^{C, \mu}(x)\right)}=R\right\}
$$

which can be seen as levels of "worst-case" convergence with rate at least $R$ for every point $x \in[a, b]$.

If an arbitrary function $f$ is analytic inside a simple, closed, and rectifiable curve $\mathcal{C}$ which is contained in a closed simply connected region around the nodes, it can be represented by the Cauchy integral formula

$$
f(x)=\frac{1}{2 \pi \mathrm{i}} \int_{\mathcal{C}} \frac{f(s)}{s-x} \mathrm{~d} s=\frac{1}{2 \pi \mathrm{i}} \int_{\mathcal{C}} f(s) g(x, s) \mathrm{d} s .
$$

The representation (11) and the linearity of the rational interpolants imply that

$$
r_{n}(x)=\frac{1}{2 \pi \mathrm{i}} \int_{\mathcal{C}} \frac{f(s)}{s-x} \cdot\left(1-\frac{\sum_{i=0}^{n-d} \lambda_{i, n}(s)}{\sum_{i=0}^{n-d} \lambda_{i, n}(x)}\right) \mathrm{d} s
$$

is the rational interpolant for $f$. The interpolation error therefore is

$$
f(x)-r_{n}(x)=\frac{1}{2 \pi \mathrm{i}} \int_{\mathcal{C}} \frac{f(s)}{s-x} \cdot \frac{\sum_{i=0}^{n-d} \lambda_{i, n}(s)}{\sum_{i=0}^{n-d} \lambda_{i, n}(x)} \mathrm{d} s,
$$

which is a Hermite-type error formula [8] for the family of rational interpolants (1). Finally,

$$
\left\|f-r_{n}\right\|_{\infty} \leq D \frac{\sup _{s \in \mathcal{C}}\left|\sum_{i=0}^{n-d} \lambda_{i, n}(s)\right|}{\min _{x \in[a, b]}\left|\sum_{i=0}^{n-d} \lambda_{i, n}(x)\right|},
$$

where $D=\frac{\text { length }(\mathcal{C}) \max _{s \in \mathcal{C}}|f(s)|}{2 \pi \operatorname{dist}([a, b], \mathcal{C})}$ is a constant independent of $n$. We summarize the above expansion in the following main theorem.

Theorem 2 Let $f$ be a function analytic in an open neighborhood of $[a, b]$, and let $R>0$ be the smallest number such that $f$ is analytic in the interior of $\mathcal{C}_{R}$ defined in (14). Then the rational interpolants $r_{n}$ defined by (2), with limiting node measure $\mu$ and $d(n) / n \rightarrow C$, satisfy

$$
\limsup _{n \rightarrow \infty}\left\|f-r_{n}\right\|_{\infty}^{1 / n} \leq R
$$



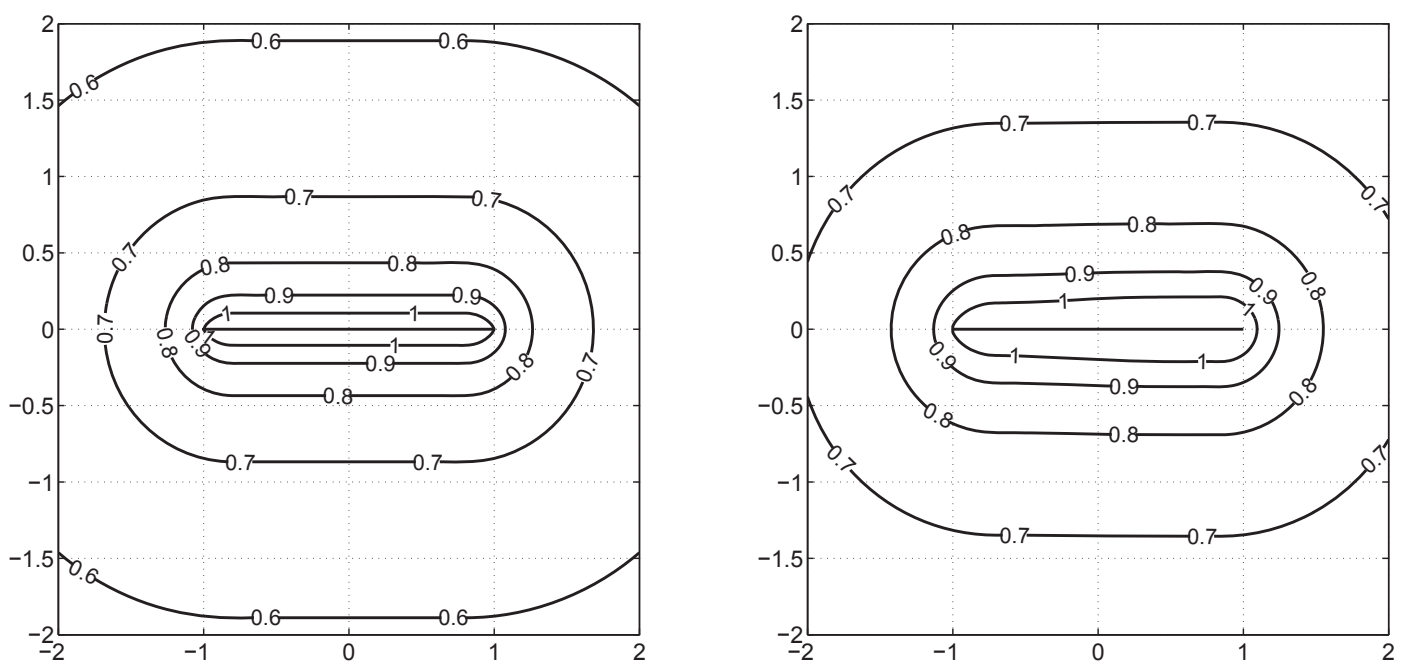

Figure 3: Level lines of convergence for barycentric rational interpolation for $C=0.2$ in equispaced nodes (left) and nodes distributed according to the density $\phi(x)=(4+$ $\arctan (4 x)) / 8$ (right) on $[-1,1]$.

In Figure 3 we illustrate the level lines $\mathcal{C}_{R}$ for the parameter $C=0.2$ with equispaced nodes on the left and with nodes distributed according to the density $\mathrm{d} \mu / \mathrm{d} x=\phi(x)=$ $(4+\arctan (4 x)) / 8$ on the right. The interval is $[-1,1]$. In section 2.3 we give more details on how we computed these level lines in the case of equispaced nodes; for arbitrary node densities we integrated the potential function $V^{C, \mu}$ numerically. For equispaced nodes, a line corresponding to $R=1$ appears close to the interval, which means that $r_{n}$ might not converge throughout $[a, b]$ to a function $f$ with a singularity $s$ in the interior of that curve. Runge's phenomenon is therefore likely to appear for such a function if $d$ increases with $n$. An intuitive explanation of why there is no Runge phenomenon with $d$ fixed goes as follows: The interpolation error may be written as

$$
f(x)-r_{n}(x)=\frac{\sum_{i=0}^{n-d} \lambda_{i, n}(x)\left(f(x)-p_{i, n}(x)\right)}{\sum_{i=0}^{n-d} \lambda_{i, n}(x)}
$$

(see [11]) and is a blend of an increasing number of polynomial interpolation errors as $n$ increases. The polynomials $p_{i, n}$ have fixed maximal degree $d$ and interpolate $f$ in subintervals $\left[x_{i, n}, x_{i+d, n}\right]$ of decreasing length, so that the region where $f$ needs to be analytic shrinks.

Theorem 2 gives only an asymptotic upper bound on the rate of convergence of $r_{n} \rightarrow f$ as $n \rightarrow \infty$, as opposed to the polynomial case, where equality holds (see Theorem 1 ). This means that, unlike the case with polynomial interpolation, we generally cannot infer the level line $\mathcal{C}_{R}$ with the closest singularity of $f$ from a known or observed approximation rate $R$. This is not a problem of our derivation, but an intrinsic property of barycentric 
rational interpolation with alternating signs in the blending functions $\lambda_{i, n}$, as we explain in the following.

\subsection{Symmetric nodes}

One reason why convergence may be faster than predicted by the asymptotic upper bound from Theorem 2 is cancellation in the terms of the error representation (12). As an illustration, we assume that the nodes $x_{i, n}$ are pairwise symmetric with respect to the midpoint $(a+b) / 2$ of the interval, i.e.,

$$
\frac{x_{i, n}+x_{n-i, n}}{2}=\frac{a+b}{2}, \quad i=0,1, \ldots, n .
$$

For these nodes, the upper bound in Lemma 2 may be crude for points $z$ relatively close to the interval and with $\operatorname{Re}(z) \in[a, b]$. This situation is illustrated in Figure 4, which shows the level lines of $\left|\sum_{i=0}^{n-d} \lambda_{i, n}(z)\right|^{1 /(n+1)}$ for equispaced nodes on $[-1,1]$ with $n=100$ and $d=9$ in the top left picture, and the levels of the asymptotic upper bound $\exp \left(V^{\mu}(z)\right)$. The level lines agree well if $\operatorname{Re}(z)$ is outside $[-1,1]$. The reason for the observed discrepancy above and below the midpoint of the interval is the fact that some terms in the sum

$$
\sum_{i=0}^{n-d(n)} \lambda_{i, n}(z)=\sum_{i=0}^{n-d(n)} \frac{(-1)^{i}}{\left(z-x_{i, n}\right) \cdots\left(z-x_{i+d(n), n}\right)}
$$

cancel mutually for certain values of $z$, or may at least be reduced. For example, the absolute value of the sum of the $\ell$ th and $(n-d(n)-\ell)$ th terms, evaluated at a point $z$ with $\operatorname{Re}(z)=(a+b) / 2$, yields after a short computation

$$
\begin{aligned}
\left|\lambda_{\ell, n}(z)+\lambda_{n-d(n)-\ell, n}(z)\right| & =\left|\lambda_{\ell, n}(z)+(-1)^{n+1} \overline{\lambda_{\ell, n}(z)}\right| \\
& = \begin{cases}2\left|\operatorname{Re}\left(\lambda_{\ell, n}(z)\right)\right| & \text { if } n \text { is odd } \\
2\left|\operatorname{Im}\left(\lambda_{\ell, n}(z)\right)\right| & \text { if } n \text { is even. }\end{cases}
\end{aligned}
$$

This simplification occurs for all $0 \leq \ell \leq\lfloor(n-d) / 2\rfloor$ and obviously reduces the interpolation error at these particular points $z$, causing the cusp in the level curves.

However, if we slightly perturb the equispaced grid, leading to almost equispaced nodes, then the levels of $\left|\sum_{i=0}^{n-d} \lambda_{i, n}(z)\right|^{1 /(n+1)}$ look much more similar to the predicted level curves $\exp \left(V^{\mu}(z)\right)$; see the bottom left picture in Figure 4. The presented convergence theory is asymptotic in the sense that we only require convergence $\mu_{n} \rightarrow \mu$ of the node measures in a weak sense. Not necessarily all the $\mu_{n}$ need to have perfectly symmetric mass points in order to satisfy this condition. It is therefore not reasonable to expect that our theory will capture the error reduction effects appearing with symmetric nodes. The difference between the levels of $\left|\sum_{i=0}^{n-d} \lambda_{i, n}(z)\right|$ for equispaced nodes and almost equispaced nodes also becomes apparent from the error curves for the interpolation of a function with a singularity inside the cusp region, say, $f(x)=1 /(x-0.3 \mathrm{i})$. This is illustrated in the bottom right picture in Figure 4. The error curves for the interpolation of $f$ for $1 \leq n \leq 150$ with 

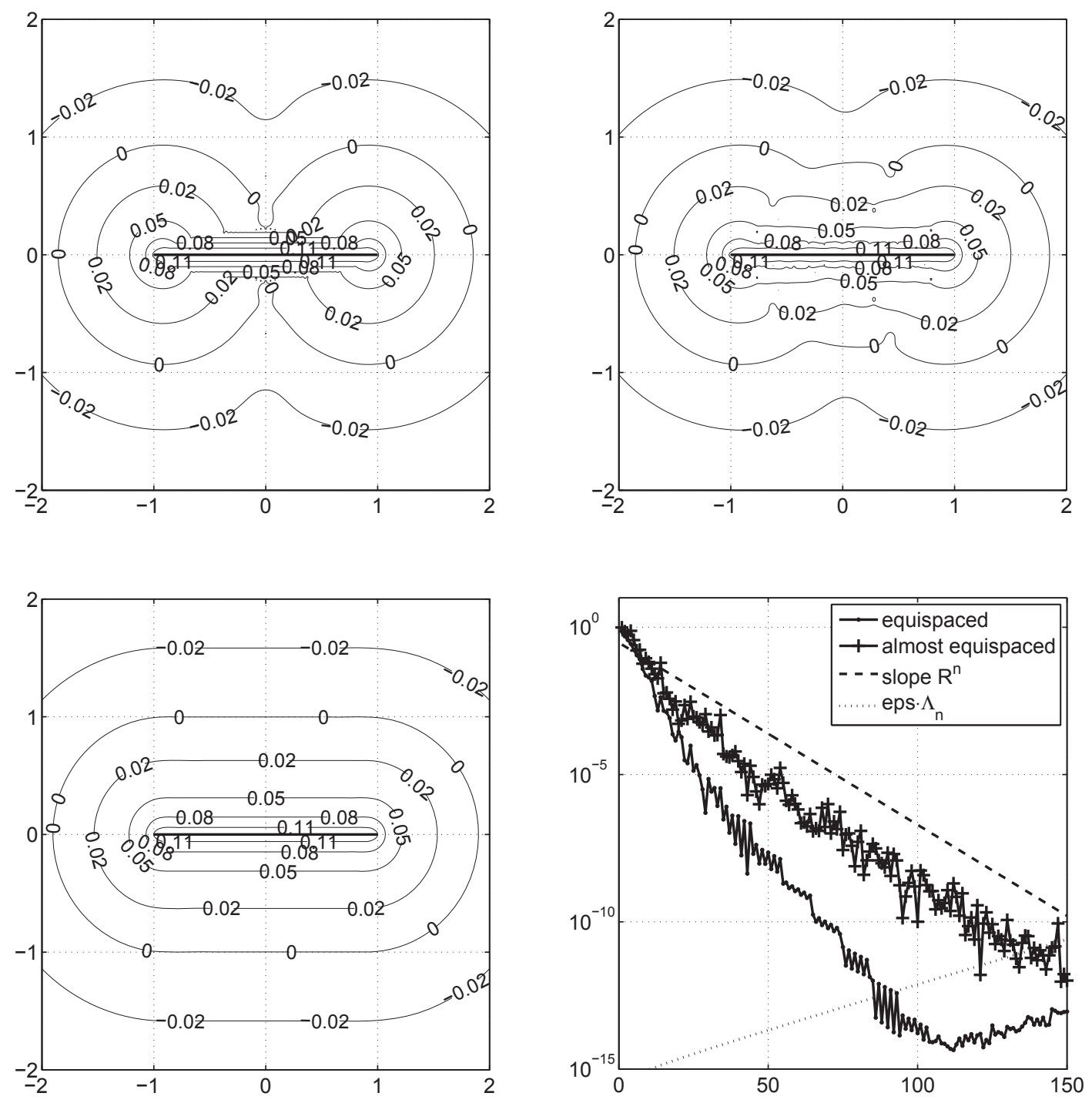

Figure 4: Top: Level lines of $\left|\sum_{i=0}^{n-d} \lambda_{i, n}(z)\right|^{1 /(n+1)}$ (left) with $d=9$ for $n=100$ equispaced nodes in $[-1,1]$ (solid central line) and level lines of $\exp \left(V^{\mu}(z)\right)$ (right) on a $\log _{10}$ scale. Bottom: Level lines for almost equispaced nodes (left). Relative error curve for the interpolation of $f(x)=1 /(x-0.3 \mathrm{i})$ with $C=0.1$ and both node sequences, asymptotic relative error bound, and upper bound on eps $\cdot \Lambda_{n}$ (right). 
$C=0.1$ and both node sequences indicate that the symmetry in equispaced nodes results in a convergence acceleration as compared to the predicted rate. The oscillating behavior of the curve corresponding to interpolation in equispaced nodes is caused by different reductions of $\left|\sum_{i=0}^{n-d} \lambda_{i, n}(z)\right|$ taking place for even and odd values of $n$; see (15).

\subsection{Equispaced nodes}

In the case of equispaced nodes $x_{i, n}$, distributed according to the density $\phi(x)=1 /(b-a)$ on $[a, b]$, we can give a more explicit statement of the rates of convergence. First, the subintervals of the local polynomial interpolants stay of constant length $C(b-a)$ as $\alpha$ varies in $[a, b-C(b-a)]$ :

$$
[\alpha, \beta(\alpha)]=[\alpha, \alpha+C(b-a)] .
$$

A formula for the potential of $\nu_{\alpha}$ is given explicitly from (6):

$$
\begin{aligned}
U^{\nu_{\alpha}}(z) & =-\int_{\alpha}^{\beta(\alpha)} \frac{1}{\beta(\alpha)-\alpha} \log |z-x| \mathrm{d} x \\
& =-\log \left(\frac{C(b-a)}{2 e}\right)-\frac{1}{2} \operatorname{Re}\left(\left(1-z^{\prime}\right) \log \left(1-z^{\prime}\right)-\left(-1-z^{\prime}\right) \log \left(-1-z^{\prime}\right)\right),
\end{aligned}
$$

where $z^{\prime}=(2 z-2 \alpha) /(C b-C a)-1$. Furthermore, the right-hand side of the inequality in Lemma 3 can be bounded easily from below for $x \in[a, b]$ : Since on the real line all the $U^{\nu_{\alpha}}$ are concave and symmetric with respect to the midpoint of the subinterval $[\alpha, \alpha+C(b-a)]$ and are simply translates of each other when $\alpha$ is varied, the minimum

$$
\min _{x \in[a, b]} \exp \left(V^{C, \mu}(x)\right)=\min _{x \in[a, b]} \max _{\alpha \in\left[a, \Phi^{-1}(1-C)\right]} \exp \left(C U^{\nu_{\alpha}}(x)\right)
$$

is obtained either for $x=a$ and $\alpha=a$ or by symmetry for $x=b$ and $\alpha=\Phi^{-1}(1-C)$. Choosing the former pair of parameters and with $x^{\prime}=-1$, it follows that

$$
U^{\nu_{a}}(a)=-\log \left(\frac{C(b-a)}{2 e}\right)-\log (2)=\log \left(\frac{e}{C(b-a)}\right),
$$

so that

$$
\min _{x \in[a, b]} \exp \left(V^{C, \mu}(x)\right)=\left(\frac{e}{C(b-a)}\right)^{C} .
$$

The contours can thus be given more explicitly as

$$
\mathcal{C}_{R}=\left\{z \in \mathbb{C}: \exp \left(V^{C, \mu}(z)\right)=R\left(\frac{e}{C(b-a)}\right)^{C}\right\} .
$$

Remark. The lower bound

$$
\min _{x \in[a, b]}\left|\sum_{i=0}^{n-d} \lambda_{i, n}(x)\right| \geq\left(\left(\frac{b-a}{n}\right)^{d+1} d !\right)^{-1}
$$


was derived in the proof of [11, Thm. 2] for the case of equispaced nodes and is valid for any set of nodes after the factor $(b-a) / n$ is replaced by $h$. This lower bound asymptotically coincides with (16): Using Stirling's approximation

$$
d ! \sim \sqrt{2 \pi d}\left(\frac{d}{e}\right)^{d}
$$

we obtain, with $d=C n$ and upon taking the $n$th root, that

$$
\left(\left(\frac{b-a}{n}\right)^{d+1} d !\right)^{-1 / n} \sim\left(\left(\frac{b-a}{n}\right)^{C n+1} \sqrt{2 \pi C n}\left(\frac{C n}{e}\right)^{C n}\right)^{-1 / n} \sim \frac{e^{C}}{C^{C}(b-a)^{C}}
$$

as $n \rightarrow \infty$.

\section{Stabilization}

In the literature there are only few recommendations for how to choose the parameter $d$ in $r_{n}$. As is suggested in [19], in practice, $d$ is typically chosen as a fixed small integer and $n$ is successively increased until the desired accuracy is achieved. Based on the asymptotic convergence theory from section 2, we may give a different recommendation for how to choose $d$ if some information on $f$ is available - for example, its region of analyticity. For simplicity of exposition, we will focus on equispaced nodes, but the same reasoning applies to arbitrary nonconstant node density functions $\phi$.

For an arbitrary distribution of interpolation nodes and $f \in C^{d+2}[a, b]$, the interpolation error decreases like $K h^{d+1}$, as explained in the introduction. Large values of $d$ should therefore lead to fast convergence as long as the function $f$ is sufficiently smooth. However, rounding errors and their amplification during the interpolation process also come into play. We thus need to address the growth of the condition number, i.e., the Lebesgue constant $\Lambda_{n}$, which increases exponentially with $d$ at least for equispaced node (see (7)) and almost equispaced nodes $[4,5]$. When $d$ grows too fast with $n$, the rounding errors might be so highly amplified that the approximation error increases again after a certain precision had been reached for relatively small $n$.

To see how the interpolation error might behave in the presence of rounding imprecision in the data, we suppose that to every function value $f_{i, n}$ is added a relative perturbation $f_{i, n} \varepsilon_{i, n}$, where every $\left|\varepsilon_{i, n}\right|$ is less than or equal to some positive $\varepsilon$. The rational interpolants of these perturbed values will be denoted by $\widetilde{r}_{n}$. As the interpolants are linear in the data, the error can be estimated as

$$
\begin{aligned}
\left\|f-\widetilde{r}_{n}\right\|_{\infty} & =\max _{a \leq x \leq b}\left|f(x)-\frac{\sum_{i=0}^{n} \frac{w_{i, n}}{x-x_{i, n}}\left(f_{i, n}+f_{i, n} \varepsilon_{i, n}\right)}{\sum_{i=0}^{n} \frac{w_{i, n}}{x-x_{i, n}}}\right| \\
& \leq\left\|f-r_{n}\right\|_{\infty}+\varepsilon \max _{a \leq x \leq b} \frac{\sum_{i=0}^{n} \frac{\left|w_{i, n}\right|}{\left|x-x_{i, n}\right|}\left|f_{i, n}\right|}{\left|\sum_{i=0}^{n} \frac{w_{i, n}}{x-x_{i, n}}\right|} \\
& \leq\left\|f-r_{n}\right\|_{\infty}+\varepsilon\|f\|_{\infty} \Lambda_{n} .
\end{aligned}
$$



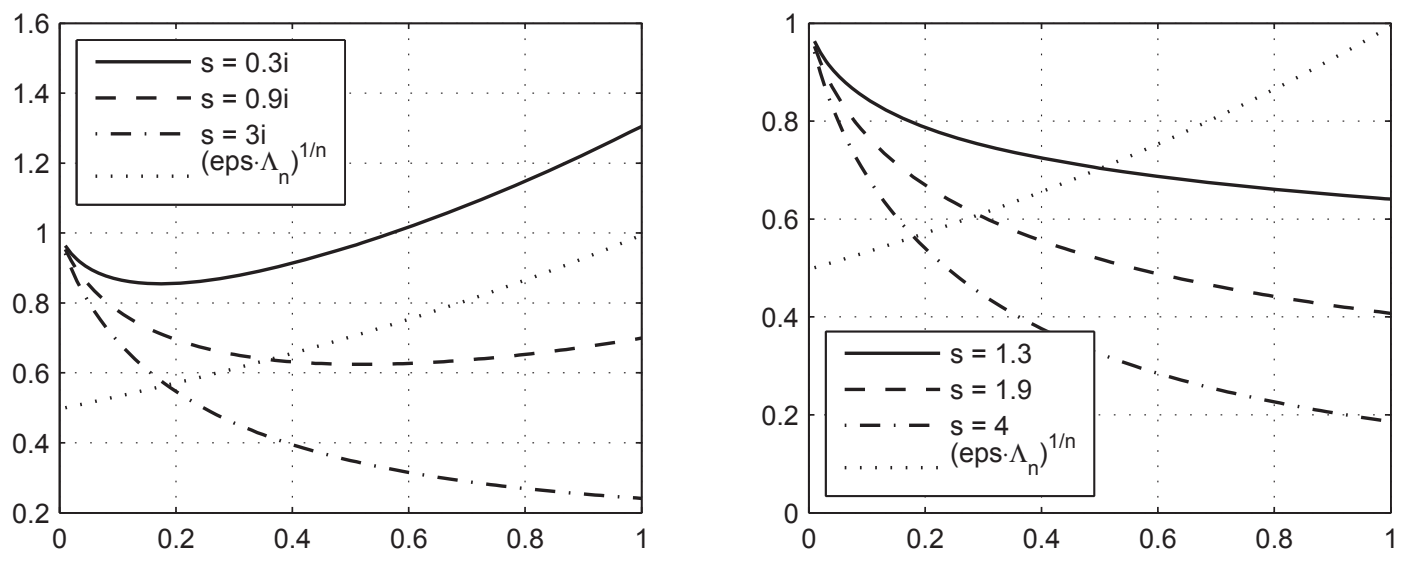

Figure 5: Convergence rates $R$ for $C$ varying in $(0,1]$ and various $s$ on the upper imaginary axis (left), and on the positive real line outside the interval $[-1,1]$ (right); the dotted lines show the upper bound on the Lebesgue constant for $n=50$.

The numerical error is thus governed by two terms - the theoretical error from exact arithmetic and the amplification of the rounding error. If $f$ satisfies the hypotheses of Theorem 2, then for $n$ large enough,

$$
\left\|f-\widetilde{r}_{n}\right\|_{\infty} \lesssim D R^{n}+\varepsilon\|f\|_{\infty} \Lambda_{n}
$$

In IEEE double precision, $\varepsilon=\mathrm{eps}=2^{-52} \approx 2.22 \cdot 10^{-16}$.

With the choice $d=\operatorname{round}(C n)$ for a fixed $C \in(0,1]$, the upper bound $(7)$ on $\Lambda_{n}$ grows at least like $2^{C n}$. This indicates that simultaneously large $C$ and $n$ are prohibitive, which equivalently corresponds to choosing a large parameter $d$ for $r_{n}$. The convergence rate $R$ in Theorem 2 also depends on $C$-however, not monotonically for every admissible singularity $s$. The solid, dashed, and dash-dot lines in Figure 5 illustrate the behavior of $R$ for $C \in(0,1]$ and various values of $s$ on the upper imaginary axis in the left picture, and positive values outside the interval $[-1,1]$ on the right. The dotted lines show the $n$th root of the upper bound (7) on the Lebesgue constant multiplied by $\varepsilon$. The maximum of the dotted line and that corresponding to a convergence rate gives a good approximation for $\left(R^{n}+\varepsilon \Lambda_{n}\right)^{1 / n}$, which can be interpreted as the observed convergence or divergence rate. We set both factors $D$ and $\|f\|_{\infty}$ to 1 since we focus on relative errors. One can see that $R$ monotonically decreases with increasing $C$ when $s$ is a real number, so that in this case it would be attractive to choose $C$ as large as possible in exact arithmetic. On the other hand, if $s$ is on the imaginary axis, then choosing too large a $C$ might result in a large $R$ as well.

Let us now investigate what a good choice of $C$ would be if we want to find a compromise between fast convergence and a reasonably small condition number. From [18] we know that no interpolation method for equispaced nodes exists that converges geometrically and whose condition number does not increase geometrically. Our aim is thus the determination 

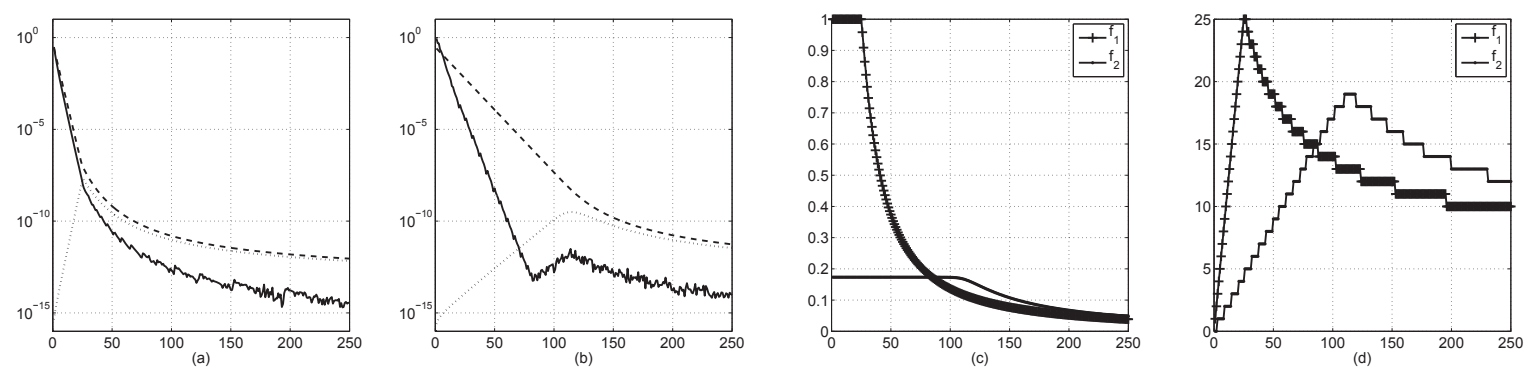

Figure 6: Relative errors (solid line) together with predicted relative error slope (dashed line) and upper bound on eps $\Lambda_{n}$ (dotted line) for the functions $f_{1}(x)=1 /(x-1.5)$ (a) and $f_{2}(x)=1 /(x-0.3 \mathrm{i})$ (b) after choosing, for each $n \in\{1, \ldots, 250\}$, the value of $C$ such that the predicted error slope is nearly minimal (c) and interpolating with $d=\operatorname{round}(C n)$ (d).

of an optimal value of $C$ for a given singularity $s$ and number of nodes $n$. This $C$ should lead to an interpolation error that is nearly as small as possible and that is not dominated by the amplification of errors in the interpolated data. Our suggestion is to minimize an upper bound on the numerical error which is a superposition of geometric convergence or divergence of the interpolant in exact arithmetic (e.a.) and the amplification of rounding errors:

observed $\operatorname{error}(C, n) \approx$ interpolation error in e.a. + imprecision $\times$ condition number

$\lesssim D\left(\exp \left(V^{C, \mu}(s)-C\right)(C(b-a))^{C}\right)^{n}$

$+\operatorname{eps} \cdot 2^{C n-1}(2+\log n)\|f\|_{\infty}$

$=$ : $\quad$ predicted error $(C, n)$.

Similar reasoning has been followed by the authors of [28]. We propose determining $C \in(0,1]$ such that the predicted error slope is nearly minimal. We performed this minimization for the functions $f_{1}(x)=1 /(x-1.5)$ and $f_{2}(x)=1 /(x-0.3 \mathrm{i})$ and display the results in Figure 6 together with the values of $C$ chosen by the minimization process for each $n$. Observe that we are minimizing only an approximate upper bound on the observed error; the convergence of the interpolation process can be faster than predicted, for instance, because of symmetry effects as described in section 2.2 or other favorable simplifications in the error term. This is also the reason for the nonmonotone error curve obtained with the interpolation of $f_{2}$. One can expect only that the observed error curve will stay (up to a constant factor) below the predicted slope. We observed that this nonmonotone behavior disappears with almost equispaced nodes, or if one heuristically chooses $D<1$ in order to take the faster convergence into account. The rightmost picture illustrates the choice of $d$ from the minimization process. For small $n, d$ may be increased quickly, but then it needs to be decreased again in order to maintain the attained precision and avoid the growth of the condition number. 
Remark. Our asymptotic upper bound on the interpolation error may be crude due to symmetry effects. However, we observe that the convergence is typically geometric with a rate $\widetilde{R} \leq R$ if $d=\operatorname{round}(C n)$, that is, $\operatorname{error}(C, n) \approx K \widetilde{R}^{n}$ with some constant $K$. In many applications, the closest singularity $s$ of $f$, in terms of level lines, is not known or difficult to determine. In such situations, interpolation is usually done for an increasing number $n$ of nodes until the approximation is accurate enough. The indication that convergence is geometric allows us to establish a heuristic method for the estimation of a value of $C$ for which the approximation error, error $(C, n)$, is below some prescribed relative tolerance reltol and $n$ is as small as possible. To this end, we assume that we have an estimator esterr $(C, n)$ for the error of the rational interpolant with $n$ nodes and parameter $d=\operatorname{round}(C n)$. Such an estimator can be obtained, e.g., from the evaluation of $\left|f(x)-r_{n}(x)\right|$ at sufficiently many points in the interval. After choosing moderate numbers $n_{1}$ and $n_{2}$ of nodes, say, $n_{1}=10$ and $n_{2}=40$, and computing esterr $\left(C, n_{1}\right)$ and esterr $\left(C, n_{2}\right)$, these estimators can be used to calculate the observed convergence or divergence rate as

$$
\widetilde{R} \approx\left(\frac{\operatorname{esterr}\left(C, n_{2}\right)}{\operatorname{esterr}\left(C, n_{1}\right)}\right)^{1 /\left(n_{2}-n_{1}\right)} .
$$

Slightly more sophisticated and robust ways of calculating $\widetilde{R}$ could certainly be derived, e.g., by taking into account more than just two values of $n$. Under the assumption that convergence is indeed geometric, in order to find a smallest possible $n$, we are interested in minimizing the rate $\widetilde{R}$ among all $C \in(0,1]$ under the constraint that the error contribution of the growing Lebesgue constant stays below reltol. In the following we sketch a simple golden-section search (see [19, Sec. 10.2]) for locally optimal $C$ and $n$ :

1. Set $C_{1}=0$ and $C_{4}=1$.

2. Set $C_{2}=\phi C_{1}+(1-\phi) C_{4}$ and $C_{3}=(1-\phi) C_{1}+\phi C_{4}$, where $\phi=\frac{\sqrt{5}-1}{2}$.

3. Compute estimates esterr $\left(C_{j}, n_{1}\right)$ and esterr $\left(C_{j}, n_{2}\right)$ of the interpolation error for $j=1, \ldots, 4$ (or reuse previously computed values).

4. Compute an estimate for the slope of convergence as

$$
\widetilde{R}_{j}=\left(\frac{\operatorname{esterr}\left(C_{j}, n_{2}\right)}{\operatorname{esterr}\left(C_{j}, n_{1}\right)}\right)^{1 /\left(n_{2}-n_{1}\right)}, \quad j=1, \ldots, 4 .
$$

5. Compute critical values $\bar{n}_{j}$ such that

$$
\widetilde{R}_{j}^{\bar{n}_{j}}=2^{C_{j} \bar{n}_{j}-1}\left(2+\log \bar{n}_{j}\right) \text { eps }, \quad j=1, \ldots, 4 .
$$

6. If $\log \left(\right.$ reltol) $/ \log \left(\widetilde{R}_{j}\right)>\bar{n}_{j}$ for some $j$, set $R_{j}=1+C_{j}$ (the desired accuracy is not attainable with this value of $C_{j}$, and hence $R_{j}$ is interpreted as divergence).

7. If $\widetilde{R}_{2} \geq \widetilde{R}_{3}$, then set $C_{1}=C_{2}$; else set $C_{4}=C_{3}$. 

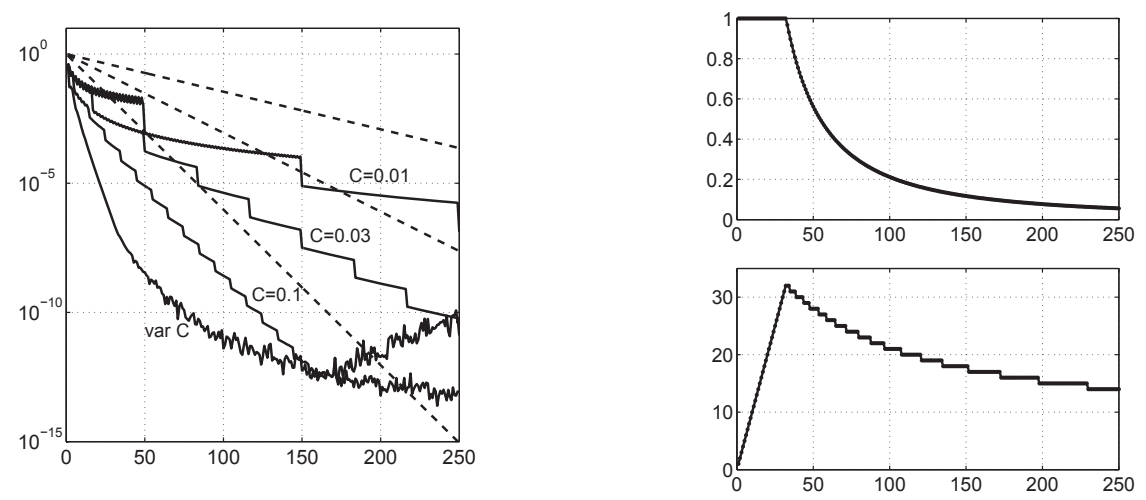

Figure 7: Relative errors for the interpolation of $f(x)=\log (1.2-x) /\left(x^{2}+2\right)$.
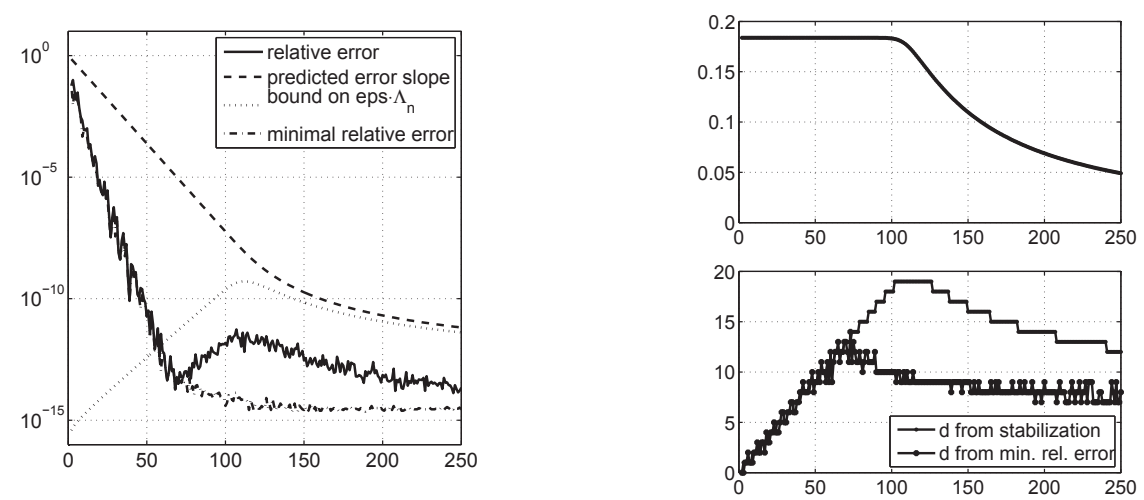

Figure 8: Relative errors for the interpolation of $f(x)=\arctan (\pi x)$.

8. If $C_{4}-C_{1}$ is larger than some tolerance (e.g., 0.01), go to Step 2.

9. Use $C_{4}$ as an approximation to the optimal $C$, and $n=\log (\operatorname{reltol}) / \log \left(\widetilde{R}_{4}\right)$.

\section{Numerical experiments}

In this section we demonstrate the convergence result from Theorem 2 and the approach for the stabilization of the rational interpolants presented in section 3. We sampled in $1 \leq n \leq 250$ points from $[-1,1]$ various functions whose regions of analyticity are known and computed the relative errors in their rational interpolants. Moreover, we computed the relative error for all admissible values of $d$ with every $n$ and kept the smallest error together with the corresponding $d$. For every example, we display, in addition to the relative interpolation error, the chosen values of $C$ and $d$ in the smaller pictures.

The first example, the interpolation of $f(x)=\log (1.2-x) /\left(x^{2}+2\right)$, illustrates the behavior of the relative interpolation error for $C$ fixed, namely, 0.01, 0.03, and 0.1 ; see Figure 7 (left). The steps in the error curves are caused by the restriction that $d$ must be 

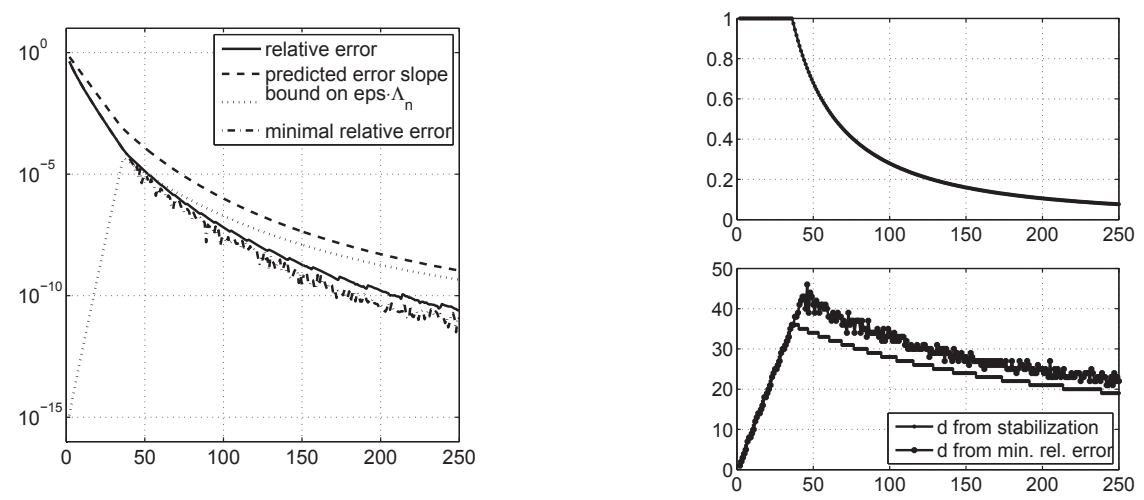

Figure 9: Relative errors for the interpolation of $f(x)=\Gamma(x+1.1)$.

an integer and every step corresponds to a change of $d$, taken as $d=\operatorname{round}(C n)$. The curve labeled $\operatorname{var} C$ is obtained by choosing $C$ and $d$ according to the minimization process. For $n \approx 150$, the value $C=0.1$ is optimal, and the corresponding curves intersect. The curve corresponding to constant $C$ increases again because of the bad conditioning with $d$ too large.

Figure 8 shows that for the interpolation of $f(x)=\arctan (\pi x)$ the error behavior is similar to that of $f_{2}(x)=1 /(x-0.3 \mathrm{i})$ in Figure 6 . Since the poles $\pm \mathrm{i} / \pi$ are too close to the interval, polynomial interpolation of this function yields Runge's phenomenon. We observed that rational interpolation with a fixed $d=6$ converges rather slowly and a relative error of $10^{-12}$ is attained with as many as $n=100$ nodes, whereas with the help of our minimization process, the latter error is already attained for $n=50$. For small $n$, our method chose near-optimal values of $d$ leading to minimal errors. However, with $n$ larger than 75 , this is not true any more. This is due to the fact that the level lines of $\left|\sum_{i=0}^{n-d} \lambda_{i}(z)\right|^{1 /(n+1)}$ with equispaced nodes yield cusps in the region where $f$ has poles; see again the top left picture in Figure 4. The speed of convergence is thus faster than predicted, and the slope of the predicted error is less steep than that of the experimental error, so that the minimization procedure does not take the growth of the Lebesgue constant into account, which leads to a recommendation of slightly too large values of $d$ compared to optimal values. This is, however, not a contradiction to our theory: The error decreased faster than predicted and therefore suffers earlier from the amplification for rounding errors measured by the Lebesgue constant. If we considerably increase the contribution of the Lebesgue constant in our minimization algorithm, then such a hump in the error curve does not show up, and the values chosen for $d$ are almost equal to those giving minimal errors. This strategy might be adopted when it is known that a function has a singularity exactly in the cusp region of the level lines describing the speed of convergence.

We investigated the same example with quasi-equispaced nodes and mesh ratio $M=3$, and we estimated the error using the bound on the Lebesgue constant associated with this kind of nodes. The resulting error follows the predicted error curve more closely. We omit the corresponding plots. We repeated a similar computation with a numerically estimated 

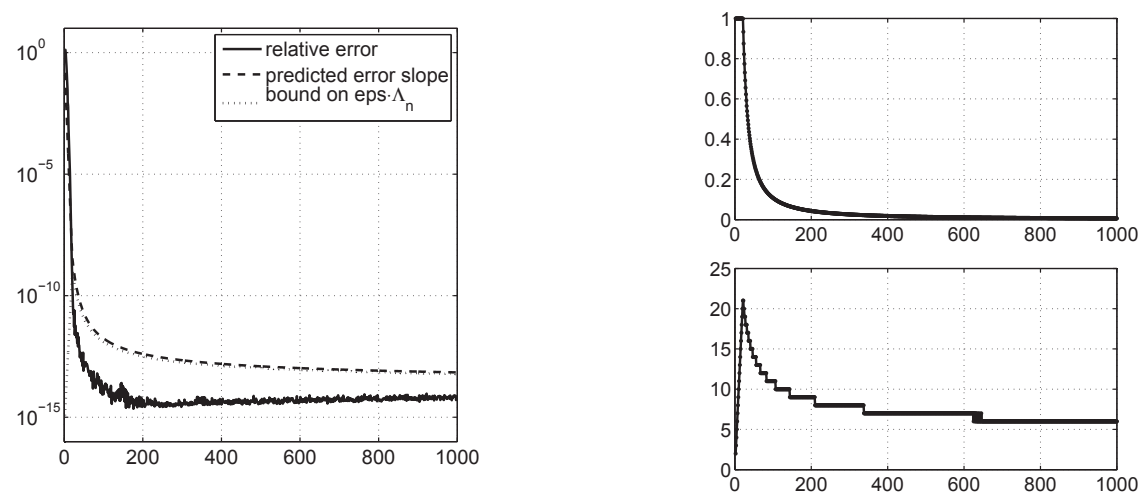

Figure 10: Relative errors for the interpolation of $f(x)=\sin (x)$ on $[-5,5]$ with $1 \leq n \leq$ 1000 .

Lebesgue constant, which again gave very similar results. Such a procedure might be used for nodes with unknown Lebesgue constant (or bounds thereof).

We also applied the minimization process to the Runge example $1 /\left(1+25 x^{2}\right)$ and observed error curves such as those in Figure 8-however, with a slightly slower convergence; a relative error of about $10^{-14}$ was observed for $n \approx 125$. This was to be expected since the poles are closer to the interval. The chosen values for $C$ did not exceed 0.12 , so that $d$ increased very slowly and decreased for $n$ larger than 200 .

The relative error in the interpolation of $f(x)=\Gamma(x+1.1)$ is plotted in Figure 9. This function has a singularity at $s=-1.1$, which is very close to the left endpoint of the interval. The convergence is therefore rather slow. In the minimization process, $d$ can thus be chosen larger, as effects of the growing Lebesgue constant become apparent only with larger $n$. Therefore, the values for $d$ chosen by our method are very close to those giving the smallest relative errors. The estimate of the condition number is slightly too large since we manipulate only upper bounds on the Lebesgue constants, which are not always very tight, and the Lebesgue constant itself already is an upper bound on the condition number.

Although the function $f(x)=\sin (x)$ is entire, we may arbitrarily take $s=10$ in our algorithm, which is sufficiently far away from the interval to guarantee fast convergence. We changed the interval to $[-5,5]$ for this example in order to compare the present results with those obtained in [11, Tab. 1] for $d=4$ constant. The authors of the latter paper tabulated absolute errors, equivalent to relative errors in this example, on the order of $10^{-12}$ for $n>600$. Figure 10 shows that an error of the same magnitude is attained for $n \approx 30$ already with the choice of $d$ of our minimization process. The chosen values for $d$ are smaller than in most of the previous examples. For this example we plotted the error for $1 \leq n \leq 1000$ to show that it remains close to machine precision even for large values of $n$. With $n$ in the tens of thousands the error did not increase significantly either.

The next demonstration concerns the heuristic for choosing "optimal" parameters $n$ and $d$ without having any information on the function $f$, as proposed in the remark of 
Table 1: Results of the algorithm described in the remark of section 3.

\begin{tabular}{|c|l|c|c|c|c|}
\hline Target relative error & Function $f$ & $C_{\text {opt }}$ & $n$ & $d$ & Observed error \\
\hline \multirow{5}{*}{$10^{-6}$} & $\log (1.2-x) /\left(x^{2}+2\right)$ & 0.61 & 40 & 24 & $5.56 \cdot 10^{-8}$ \\
& $\Gamma(x+1.1)$ & 0.42 & 75 & 32 & $5.90 \cdot 10^{-7}$ \\
& $\Gamma(x+2)$ & 0.29 & 26 & 7 & $3.66 \cdot 10^{-8}$ \\
& $\arctan (\pi x)$ & 0.19 & 31 & 6 & $2.27 \cdot 10^{-7}$ \\
& $\sin (5 x)$ & 0.33 & 22 & 7 & $4.80 \cdot 10^{-6}$ \\
\hline \multirow{5}{*}{$1^{-9}$} & $\log (1.2-x) /\left(x^{2}+2\right)$ & 0.30 & 73 & 22 & $2.36 \cdot 10^{-10}$ \\
& $\Gamma(x+1.1)$ & 0.15 & 151 & 22 & $3.06 \cdot 10^{-9}$ \\
& $\Gamma(x+2)$ & 0.29 & 39 & 11 & $2.51 \cdot 10^{-11}$ \\
& $\arctan (\pi x)$ & 0.19 & 47 & 9 & $2.47 \cdot 10^{-10}$ \\
& $\sin (5 x)$ & 0.39 & 34 & 11 & $5.50 \cdot 10^{-10}$ \\
\hline
\end{tabular}

section 3. For all functions in this section we have run this algorithm with a targeted relative error tolerance of $10^{-6}$ and $10^{-9}$, respectively. The convergence slope was estimated using interpolants with $n_{1}=10$ and $n_{2}=40$ points. The results are shown in Table 1 . They indicate that this approach can give quite good results, although a careful user would always revalidate the accuracy of the obtained interpolant $r_{n}$ by comparing $f$ and $r_{n}$ at sufficiently many points.

The last example demonstrates that the presented convergence theory is also valid for nonequispaced nodes. To this end we consider the node density $\phi(x)=(4+\arctan (4 x)) / 8$ on the interval $[-1,1]$. We have computed the nodes $x_{i, n}$ distributed according to this density by evaluating the inverse cumulative node distribution $\Phi^{-1}(i / n)$ for $i=0,1, \ldots, n$; see Figure 11 (left). The level lines with the convergence rates for $C=0.2$ are shown in Figure 3 (right). They predict the somewhat counterintuitive effect which we observe numerically in Figure 11 (right): When interpolating $f_{+}(x)=(x-1.2)^{-2}$, the convergence is slower than for $f_{-}(x)=(x+1.2)^{-2}$, although for $f_{+}$the singularity $s=1.2$ is to the right of $[-1,1]$, where the nodes are about twice as dense as on the left end of the interval. This suggests that local refinement close to a singularity may not yield the desired faster convergence; the overall node distribution $\phi$ must be taken into account.

\section{Conclusion}

We have presented a convergence theory for the family of analytic linear barycentric rational interpolants proposed by Floater and Hormann [11]. This convergence theory is valid if the blending parameter $d$ is chosen proportional to the number of nodes, and gives an upper bound on the asymptotic convergence rate when interpolating analytic functions. Our theory contains that of polynomial interpolants as a special case. Based on this theory we gave recommendations of how to choose the involved parameters heuristically in a nearoptimal way, balancing a best possible convergence rate with a controlled growth of the condition number, i.e., the Lebesgue constant associated with the interpolants. Typically, 

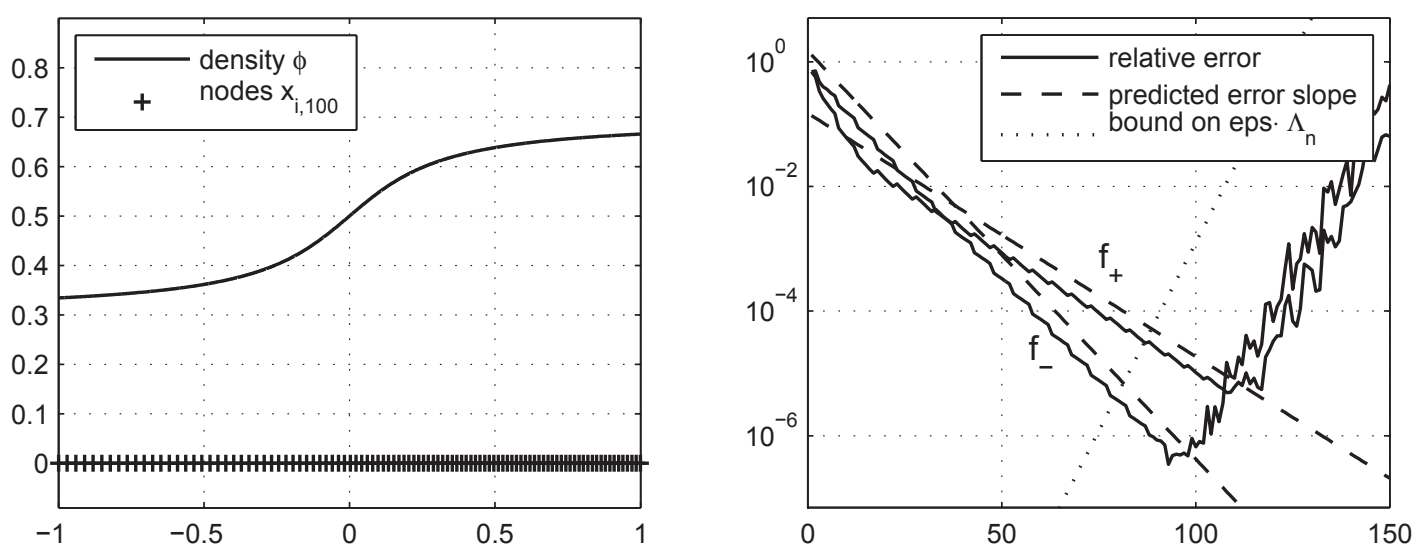

Figure 11: Nonequispaced nodes with their density $\phi(x)=(4+\arctan (4 x)) / 8$ on $[-1,1]$ (left) and convergence of the interpolation in these nodes for $f_{-}$and $f_{+}$with singularity $s=-1.2$ and $s=1.2$, respectively, together with the predicted error slope $0.860^{n}$ and $0.914^{n}$, respectively, for $1 \leq n \leq 150$ (right).

our heuristic algorithms choose the parameter $d$ as large as possible, but not too large, to ensure that the amplification of rounding errors, measured by the associated Lebesgue constant, is below the accuracy of the interpolant. Numerical tests indicate that this approach allows for a quite robust "black-box" construction of linear rational interpolants in the fewest possible nodes.

We hope that our results enhance the understanding of the convergence behavior of linear barycentric interpolation and that they make it possible to compare such interpolation schemes with other available methods for interpolation in equispaced nodes, such as the approach proposed in [14] and in the references therein, that in [15], or the schemes reviewed and cited in [18].

\section{Acknowledgments}

The authors thank Jean-Paul Berrut and Nick Trefethen for their advice and hospitality and the anonymous referees for reviewing and commenting on the paper.

\section{References}

[1] R. Baltensperger, J.-P. Berrut, and B. Noël. Exponential convergence of a linear rational interpolant between transformed Chebyshev points. Math. Comp., 68:11091120, 1999.

[2] J.-P. Berrut. Rational functions for guaranteed and experimentally well-conditioned global interpolation. Comput. Math. Appl., 15:1-16, 1988. 
[3] J.-P. Berrut and L. N. Trefethen. Barycentric Lagrange interpolation. SIAM Rev., 46:501-517, 2004.

[4] L. Bos, S. De Marchi, and K. Hormann. On the Lebesgue constant of Berrut's rational interpolant at equidistant nodes. J. Comput. Appl. Math., 236:504-510, 2011.

[5] L. Bos, S. De Marchi, K. Hormann, and G. Klein. On the Lebesgue constant of barycentric rational interpolation at equidistant nodes. Numer. Math., 121:461-471, 2012.

[6] L. Bos, S. De Marchi, K. Hormann, and S. Sidon. Bounding the Lebesgue constant for Berrut's rational interpolant at general nodes. J. Approx. Theory. Submitted.

[7] L. Brutman. Lebesgue functions for polynomial interpolation-a survey. Ann. Numer. Math., 4:111-127, 1997.

[8] P. J. Davis. Interpolation and Approximation. Dover, New York, 1975.

[9] H. Ehlich and K. Zeller. Auswertung der Normen von Interpolationsoperatoren. Math. Ann., 164:105-112, 1966.

[10] J. F. Epperson. On the Runge example. Amer. Math. Monthly, 94:329-341, 1987.

[11] M. S. Floater and K. Hormann. Barycentric rational interpolation with no poles and high rates of approximation. Numer. Math., 107:315-331, 2007.

[12] B. Fornberg. A Practical Guide to Pseudospectral Methods. Cambridge University Press, Cambridge, UK, 1996.

[13] K. Hormann, G. Klein, and S. De Marchi. Barycentric rational interpolation at quasiequidistant nodes. Dolomites Res. Notes Approx., 5:1-6, 2012.

[14] D. Huybrechs. On the Fourier extension of nonperiodic functions. SIAM J. Numer. Anal., 47:4326-4355, 2010.

[15] G. Klein. An extension of the Floater-Hormann family of barycentric rational interpolants. Math. Comp. to appear.

[16] R. B. Platte. How fast do radial basis function interpolants of analytic functions converge? IMA J. Numer. Anal., 31:1578-1597, 2011.

[17] R. B. Platte and T. A. Driscoll. Polynomials and potential theory for Gaussian radial basis function interpolation. SIAM J. Numer. Anal., 43:750-766, 2005.

[18] R. B. Platte, L. N. Trefethen, and A. B. J. Kuijlaars. Impossibility of fast stable approximation of analytic functions from equispaced samples. SIAM Rev., 53:308318, 2011. 
[19] W. H. Press, S. A. Teukolsky, W. T. Vetterling, and B. P. Flannery. Numerical Recipes: The Art of Scientific Computing, 3rd ed. Cambridge University Press, Cambridge, UK, 2007.

[20] T. Ransford. Potential Theory in the Complex Plane. Cambridge University Press, Cambridge, UK, 1995.

[21] C. Runge. Über empirische Funktionen und die Interpolation zwischen äquidistanten Ordinaten. Zeit. Math. Phys., 46:224-243, 1901.

[22] E. B. Saff and V. Totik. Logarithmic Potentials with External Fields. Springer, Berlin, 1997.

[23] A. Schönhage. Fehlerfortpflanzung bei Interpolation. Numer. Math., 3:62-71, 1961.

[24] S. J. Smith. Lebesgue constants in polynomial interpolation. Annales Math. Inf., 33:109-123, 2006.

[25] H. Stahl. Convergence of rational interpolants. Bull. Belg. Math. Soc. Simon Stevin, 3:11-32, 1996.

[26] L. N. Trefethen. Computing numerically with functions instead of numbers. Math. Comput. Sci., 1:9-19, 2007.

[27] L. N. Trefethen et al. Chebfun Version 4.0. The Chebfun Development Team, 2011. http://www.maths.ox.ac.uk/chebfun/.

[28] L. N. Trefethen and J. A. C. Weideman. Two results on polynomial interpolation in equally spaced points. J. Approx. Theory, 65:247-260, 1991.

[29] A. H. Turetskii. The bounding of polynomials prescribed at equally distributed points. Proc. Pedag. Inst. Vitebsk, 3:117-127, 1940.

[30] H. Wallin. Potential theory and approximation of analytic functions by rational interpolation. In: Proceedings of the Colloquium on Complex Analysis at Joensuu, Lecture Notes in Math., Springer-Verlag, Berlin, 1979, 434-450.

[31] J. L. Walsh. Interpolation and Approximation by Rational Functions in the Complex Domain, 5th ed. AMS, Providence, RI, 1969. 